\title{
A minor perimesencephalic subarachnoid hemorrhage on CT changes
}

\author{
Yeting Zhou' ${ }^{1}$, Daoming Tong ${ }^{2}$
}

${ }^{1}$ Medical Evaluation Unit, Shuyang People’Hospital, Shuyang, China;

${ }^{2}$ Departments of Neurology, Shuyang People'Hospital, Shuyang, China.

Email: zyt5099@163.com

Received 2 July 2011; revised 25 July 2011; accepted 4 August 2011.

\begin{abstract}
Despite the increasing number of reports of patients with perimesencephalic subarachnoid hemorrhage (PMSAH), a minor or atypical PMSAH on CT changes has not been reported. We present the first described case of a minor subarachnoid hemorrhage located in the right perimesencephalic cistern on CT $4 \mathrm{~h}$ after headache onset. Twenty-six hours after headache onset, another CT of the head showed that blood dispersion. On the third day in hospital, examination of cerebrospinal fluid revealed xanthochromia. Minor PMSAH is rare type of SAH, and can been missed if there is a delay in CT imaging of the head. Any patient with a suspected minor PMSAH or equivocal results on CT should undergo routine lumbar puncture.
\end{abstract}

Keywords: Headache; Perimesencephalic Subarachnoid Hemorrhage; CT Imaging; Lumbar Puncture;

Angiography

\section{INTRODUCTION}

Perimesencephalic subarachnoid hemorrhage (PMSAH) appears to have an etiology and natural history distinct from aneurysm rupture [1].

The radiographic pattern of PMSAH is relatively distinct. It involves hemorrhage centered anterior to the midbrain or pons (with or without extension of blood around the brainstem) into the suprasellar cistern, or into the proximal Sylvian fissure [2]. Headache from PMSAH is a common presenting chief complaint. Despite the increasing number of reports of patients with PMSAH [1, $3,4]$, a minor or atypical PMSAH has not been reported. Here, we detail the rare case of CT changes in the brain of a patient with headache from minor PMSAH.

\section{CASE REPORT}

A 36-year-old female was admitted to an affiliated hos- pital of university because of headache. Five hours before hospital admission, she suddenly began to have a headache without nausea or vomiting. Four hours after the onset of headache, CT of the head revealed a minor or equivocal subarachnoid hemorrhage (SAH) located in the right perimesencephalic cistern (Figures 1(a) and (b)).

On physical examination, the blood pressure was 150/ $72 \mathrm{mmHg}$. She was alert and oriented, with mild nuchal rigidity. The results of the remainder of the physical examination were normal. Laboratory examinations, including complete blood count, serum electrolytes, liver

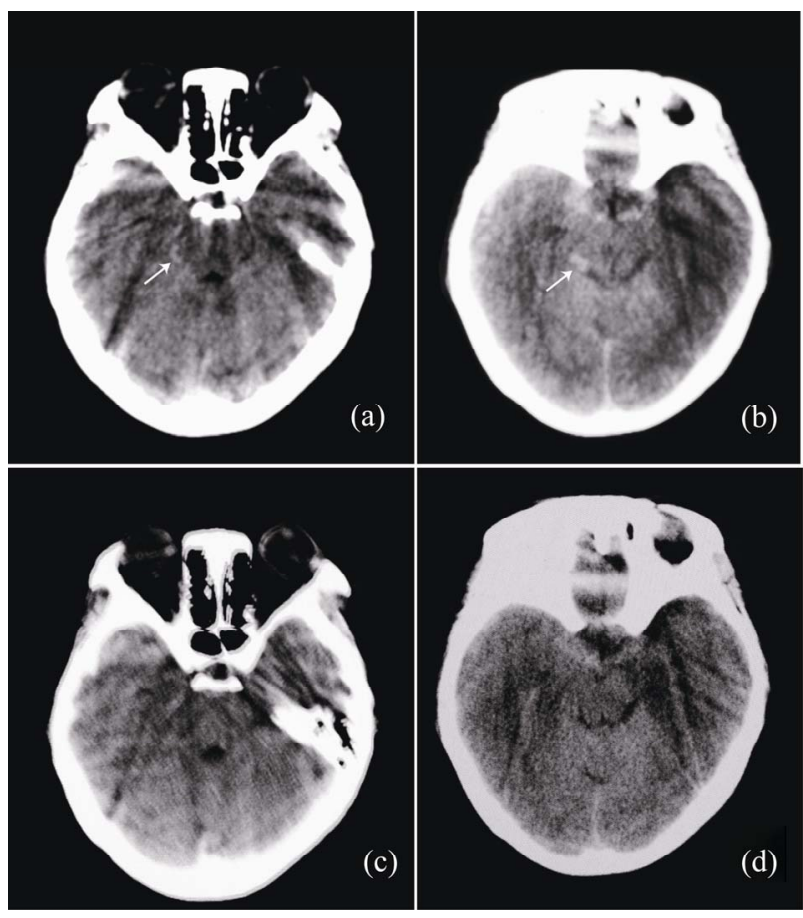

Figure 1. Four hours after headache onset, CT of the head revealed a minor or equivocal subarachnoid hemorrhage ((a) and (b), arrow). Twenty-six hours after headache onset, CT of the head showed that extravasated blood dispersed quickly ((c) and (d)). 
function test, and coagulation studies were all normal. A diagnosis of SAH was suspected (Hunt-Hess grade I grade).

Twenty-six hours after the onset of headache, CT of the head showed that extravasated blood dispersed quickly (Figures 1(c) and (d)). On the third day of hospital admission, examination of cerebrospinal fluid (CSF) revealed xanthochromia, and cytologic examination of CSF showed metemoglobin; these are characteristic features of subarachnoid hemorrhage. On the fifth day of hospital admission, digital subtraction cerebral angiography did not demonstrate an aneurysm stemming from the basilar, anterior cerebral, middle cerebral or posterior cerebral arteries. The patient reported no specific risk factors for arterial dissection (e.g., trauma) and had no significant medical history of hypertension. We made a diagnosis of minor PMSAH.

The patient was treated with intravenous anti-fibrinolytic agents. Within 3 days her symptoms and signs had resolved. She was discharged home in excellent condition 2 weeks after hospital admission. No further episodes occurred during the subsequent 12-month followup.

\section{CONCLUSION}

PMSAH is diagnosed on the basis of CT or MRI. These imaging methods demonstrate a localized area of hemorrhage centered within the perimesencephalic or prepontine cisterns without intracerebral or intraventricular extension [1,3]. Very few reports describe CT changes of minor or atypical PMSAH.

Currently our patient, within $4 \mathrm{~h}$ after the onset of a sudden headache, a minor or equivocal area of hemorrhage in the right perimesencephalic cistern was seen on head CT. This suggested the possibility of minor PMSAH. Twenty-six hours after headache onset, CT of the head showed that extravasated blood had dispersed quickly. On the third day of hospital admission, our patient revealed xanthochromia by lumbar puncture, and cytologic examination of CSF showed metemoglobin. A minor PMSAH was demonstrated.

PMSAHs account for $~ 5 \%$ of all cases of subarachnoid hemorrhage [5]. However, in our case, a typical PMSAH was not revealed on head CT $26 \mathrm{~h}$ after headache onset. Hence, the diagnosis of minor PMSAH would have been missed if our patient with this condition had not presented to a hospital or delayed in the time of CT scan.

CT may be less sensitive in patients with a SAH presenting with "minor leaks" or normal neurological status [6]. A diagnosis of SAH with a negative CT of the head accounts for $7 \%$ of cases with SAH [7]. Therefore, we speculated that the true prevalence of PMSAH may be
$>5 \%$. This also suggests that some patients with a $\mathrm{SAH}$ with a negative finding on head CT may have a minor PMSAH. One explanation of this finding is that some patients have only a small amount of blood on initial CT imaging and, in some patients, the extravasation might be too small to be detected [6]. The present study showed that a low grade on the Hunt-Hess scale, normal diastolic blood pressure, and a delay in the time of CT imaging after hospital admission could be the cause of SAH with negative findings on head CT [8]. Lumbar puncture should therefore be carried out in any patient with suspected SAH and negative (or equivocal) results on head CT [9].

Studies indicate that most of the causes in patients with PMSAH are non-aneurysmal [3,4], but some can also be aneurismal $[1,3,10]$. In our patient, diagnostic cerebral angiography did not reveal an aneurysm, but aneurysmal PMSAH was not excluded. Minor PMSAH is rare. Our case did not reveal typical PMSAH on CT. It also serves as a reminder of the importance of lumbar puncture and the variability of angiographic results displayed by PMSAH. PMSAH accounts for approximately one-third of cases of non-aneurysmal SAH [5]. The remaining cases of non-aneurysmal SAH are heterogeneous, including SAH caused by vascular malformations, venous bleeding [11], and not confined to cases of idiopathic SAH [5], Aneurysm rupture occasionally produces a PMSAH-like pattern of bleeding [10,12], In a review of 3 large series concerning the neurological complications associated with DSA, the complication rate for patients with $\mathrm{SAH}$ was $0.25 \%$ [13], so studies recommemded that $\mathrm{CT}$ angiography only is the most beneficial approach in patients with a perimesencephalic hemorrhage on CT, and accurately excludes and detects vertebrobasilar aneurysms [12,14].

In conclusion, minor PMSAH is rare type of $\mathrm{SAH}$, and can been missed if there is a delay in CT imaging of the head. Any patient with a suspected minor PMSAH or equivocal results on CT should undergo routine lumbar puncture.

\section{REFERENCES}

[1] Rinkel, G.J.E., Wijdicks, E.F.M., Hasan, D., et al. (1991) Outcome in patients with subarachnoid haemorrhage and negative angiography according to pattern of haemorrhage on computed tomography. Lancet, 338, 964-968. doi:10.1016/0140-6736(91)91836-J

[2] Schwartz, T.H. and Solomon, R.A. (1996) Perimesencephalic nonaneurysmal subarachnoid hemorrhage: Review of the literature. Neurosurgery, 39, 433-440.

[3] Van Gijn, J., Van Dongen, K.J., Vermeulen, M., et al. (1985) Perimesencephalic hemorrhage: A nonaneurysmal and benign form of subarachnoid hemorrhage. Neurology, 35, 493-497. 
[4] Hui, F.K., Tumialán, L.M., Tanaka, T., et al. (2009) Clinical differences between angiographically negative, diffuse subarachnoid hemorrhage and perimesencephalic subarachnoid hemorrhage. Neurocrit Care, 11, 64-70. doi:10.1007/s12028-009-9203-2

[5] Flaherty, M.L., Haverbusch, M., Kissela, B., et al. (2005) Perimesencephalic subarachnoid hemorrhage: Incidence, risk factors, and outcoma. Journal of Stroke Cerebrovascular Diseases, 14, 267-71. doi:10.1016/j.jstrokecerebrovasdis.2005.07.004

[6] Van der Wee, N., Rinkel, G.J.E., Hasan, D., et al. (1995) Detection of subarachnoid haemorrhage on early CT: Is lumbar puncture still needed after a negative scan? Journal of Neurology, Neurosurgery \& Psychiatry, 58, 357359. doi:10.1136/jnnp.58.3.357

[7] Richard, L.B, William, R.M, Natalie, S., Gelareh, Z.G., Shanna, F. and Larry, J.B. (2008) Sensitivity of noncontrast cranial computed tomography for the emergency department diagnosis of subarachnoid hemorrhage. Annals of Emergency Medicine, 51, 697-703. doi:10.1016/j.annemergmed.2007.10.007

[8] Tong, D.M. and Chou, Y.T. (2010) Predictors of the subarachnoid hemorrhage of a negative CT scan. Stroke, 41, e566-e567. doi:10.1161/STROKEAHA.109.574038
[9] Suarez, J.I., Tarr, R.W. and Selman, W.R. (2006) Aneurysmal subarachnoid hemorrhage. New England Journal of Medicine, 354, 387-396. doi:10.1056/NEJMra052732

[10] Schievink, W.I., Wijdicks, E.F.M., Piepgras, D.G., et al. (1994) Perimesencephalic subarachnoid hemorrhage: Additional perspectives from four cases. Stroke, 25, 1507-1511. doi:10.1161/01.STR.25.7.1507

[11] Van Der Schaaf, I.C., Velthuis, B.K., Gouw, A., et al. (2004) Venous drainage in perimesencephalic hemorrhage. Stroke, 35, 1614-1618. doi:10.1161/01.STR.0000131657.08655.ce

[12] Velthuis, B.K., Rinkel, G.J., Ramos, L.M., et al. (1999) Perimesencephalic hemorrhage: Exclusion of vertebrobasilar aneurysms with CT angiography. Stroke, 30, 1103-1109. doi:10.1161/01.STR.30.5.1103

[13] Cloft, H.J., Joseph, G.J. and Dion, J.E. (1999) Risk of cerebral angiography in patients with subarachnoid hemorrhage, cerebral aneurysm, and arteriovenous malformation: A meta-analysis. Stroke, 30, 317-320. doi:10.1161/01.STR.30.2.317

[14] Ruigrok, Y.M., Rinkel, G.J., Buskens, E., et al. (2000) Perimesencephalic hemorrhage and CT angiography: A decision analysis. Stroke, 31, 2976-2983. doi:10.1161/01.STR.31.12.2976 